\title{
ESTUDO DE CONFIABILIDADE DE VIGAS DE CONCRETO PROTENDIDO
}

\author{
Rafael Gonzales Rocha ${ }^{1}$ \\ Mauro de Vasconcellos Real ${ }^{2}$ \\ Márcio Wrague Moura ${ }^{3}$
}

\begin{abstract}
Resumo: O objetivo do presente trabalho é avaliar a confiabilidade estrutural nos projetos de vigas portuárias de concreto protendido, conforme os critérios da NBR 6118/2014. O estudo é aplicado ao projeto de vigas protendidas de estruturas portuárias, devido à necessidade de se verificar a influência da variação dos parâmetros de projeto sobre a segurança da estrutura. Será verificada a confiabilidade em relação ao estado limite último de flexão.Inicialmente desenvolveu-se um modelo para análise de estruturas portuárias de concreto, aplicável a seções transversais retangulares e "T", tal modelo foi validado confrontando os resultados obtidos com dados experimentais pré-existentes. Posteriormente procedeu-se a avaliação da probabilidade de falha segundo o método de confiabilidade de primeira ordem (FORM - First Order Reliability Method). Para avaliação da confiabilidade utilizam-se rotinas computacionais com base nos estudos de Low e Tang (2008).Depois de calculado o índice de confiabilidade foram realizados estudos paramétricos com vistas a observar a variação deste frente a três variáveis aleatórias: resistência característica à compressão do concreto; resistência característica de ruptura por tração da armadura ativa; relação entre carga permanente e carga variável. Este estudo permite estimar a sensibilidade do projeto em relação às variáveis aleatórias consideradas no modelo. Em função dos dados observados verificou-se que a maior variação no índice de confiabilidade ocorreu frente à variação da relação entre carga permanente e carga total, porém, destaca-se a importância da análise conjunta das variáveis envolvidas no processo, bem como a relevância de uma análise detalhada da confiabilidade frente à variação da resistência característica de ruptura a tração da armadura ativa.
\end{abstract}

Palavras-chave: estruturas portuárias, concreto protendido, confiabilidade, FORM.

Abstract: The objective of this study is to evaluate the structural reliability in the design of port prestressed concrete beams, according to the criteria of NBR 6118/2014. The study is applied to the design of port prestressed beams to verify the influence of the variation of design parameters on the structural safety. The reliability will be checked against the ultimate limit state in flexure. Initially, it was developed a model for the analysis of port concrete structures, that was applied to rectangular and " $\mathrm{T}$ " cross sections. The model was validated by comparing its results with pre-existing experimental data. Subsequently, it was carried out the evaluation of the probability of failure, or the equivalent reliability index, by using the First Order Reliability Method (FORM).Computational routines, based on studies of Low and Tang (2008), were used for reliability assessment.Parametric studies were performed to observe the influence of the three main random variables: characteristic compressive strength of concrete; characteristic tensile strength strength of the prestressed reinforcement; and the ratio between dead load and live load. This study allows us to estimate the sensitivity of the project safety with respect to the random variables considered in the model. According to the observed data, it was found that the greatest variation in the reliability index was due to the variation of the ratio between dead load and live load. However, it is noteworthy the importance of a joint analysis of the variables involved in the process as well as the relevance of a detailed analysis of the variation of the

\footnotetext{
${ }^{1}$ Universidade Federal do Rio Grande - FURG. E-mail: rafaelgonzales@ furg.br

${ }^{2}$ PPGEO, Programa de Pós-Graduação em Engenharia Oceânica, Universidade Federal do Rio Grande FURG. E-mail: mauroreal@ furg.br

${ }^{3}$ EE, Escola de Engenharia, Universidade Federal do Rio Grande - FURG. E-mail: mwmoura@ furg.br
} 
characteristic tensile strength of the prestressed reinforcement.

Keywords: port structures, prestressed concrete, reliability, FORM.

\section{INTRODUÇÃO}

As estruturas portuárias têm grande importância na logística de transportes utilizada mundialmente, visto que, o transporte hidroviário escoa grande parte da produção dos países, interna e externamente, necessitando para tanto de portos com grande capacidade estrutural.

A grande variabilidade nas características dos materiais componentes do concreto, bem como a alta variação da sua resistência, tornam de suma importância à aplicação de metodologias estatísticas na avaliação das estruturas.

O objetivo do estudo apresentado neste artigo é a avaliaçãoda confiabilidade estrutural de vigas portuárias de concreto protendido frente ao estado limite último de flexão, utilizando os critérios de análise e dimensionamento presentes na NBR 6118/2014.Este estudo compreendeu o desenvolvimento de rotinas computacionais para a análise estrutural das vigas portuárias e posteriormente a avaliação do índice deconfiabilidade com aplicação do método FORM.

Devido à exposição a diversos fatores climáticos, ambiente altamente agressivo, e ainda, ação de grandes carregamentos externos, as estruturas portuárias demandam alto rigor em relação à segurança estrutural.Sabe-se que, quanto mais robusta uma estrutura maior o custo empregado na sua execução, ou seja, uma estrutura 99\% segura, possui custo mais elevado que uma estrutura 95\% segura.Sendo assim, se faz necessária uma análise visando conciliar segurança e economicidade.

Diante das elevadas cargas aplicadas às estruturas portuárias e da necessidade de reduzir a abertura de fissuras, devido à agressividade do ambiente, a aplicação do concreto protendido nos elementos constitutivos destas estruturas, principalmente em vigas, torna-se indispensável. Com a utilização do concreto protendido objetiva-se transpor grandes vãos, suportar grandes cargas e aumentar a vida útil da estrutura (limitando a ocorrência de fissuras).

Nas figuras de 1 a 4 são apresentados exemplos de estruturas de concreto protendido utilizadas na construção de um cais de porto.

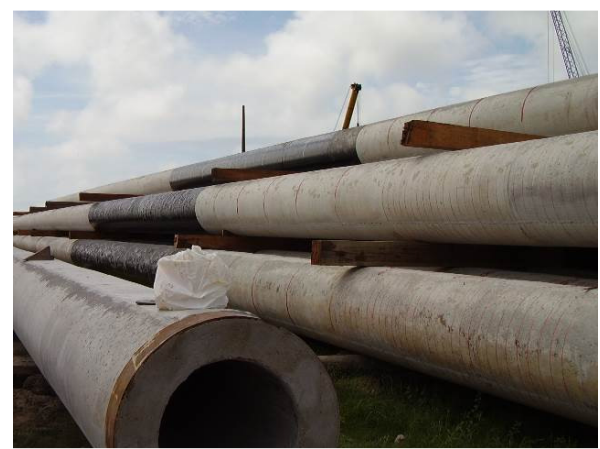

Figura 1- Estacas pré-moldadas protendidas (estocagem) -TECON/RGRS.

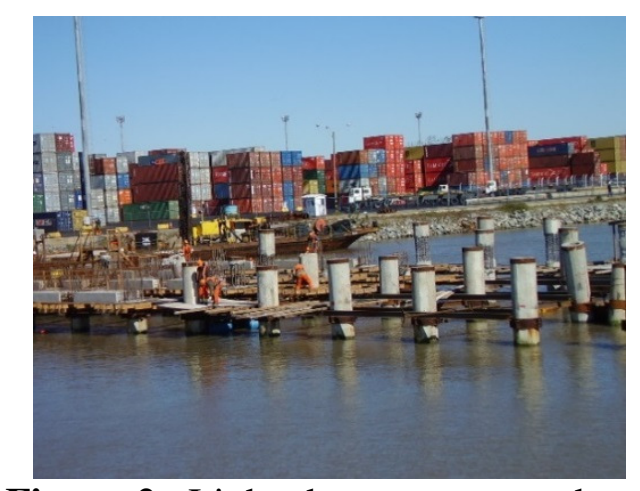

Figura 2- Linha de estacas cravadas TECON/RG-RS.

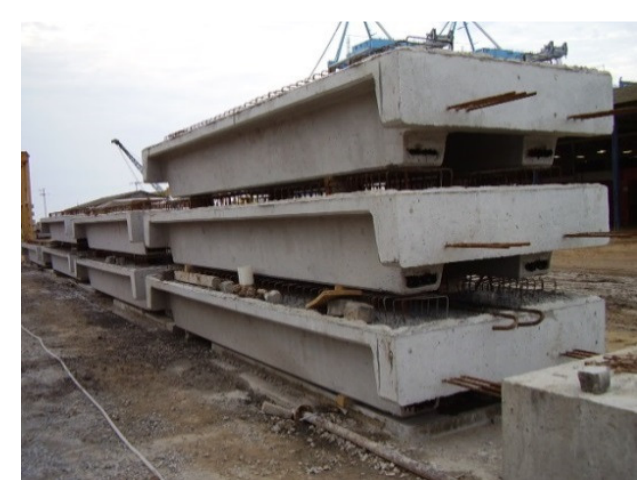

Figura 3- Vigas pré-moldadas protendidas tipo $\pi$ (estocagem) TECON/RG. 


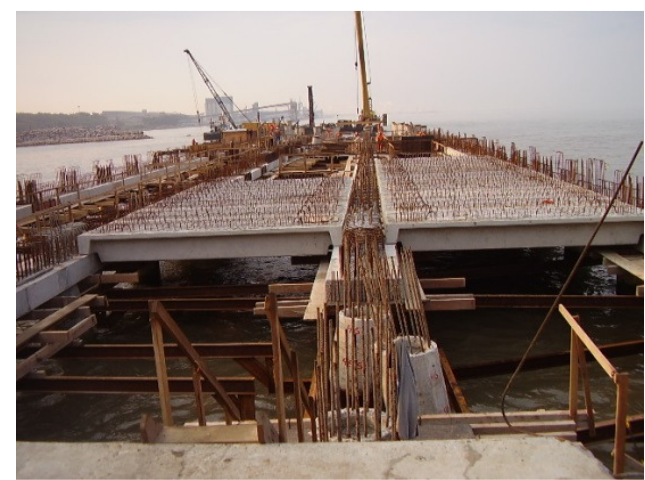

Figura 4- Vigas pré-moldadas

protendidas tipo $\pi$ (montagem do cais) TECON/RG.

Os projetos estruturais devem considerar incertezas associadas às propriedades dos materiais, às propriedades geométricas e aos carregamentos. Essas incertezas tornam impossível a elaboração de um projeto que apresente segurança total ou probabilidade de falha nula, visto que, determinada combinação de valores das variáveis pode resultar em uma condição de falha. Com intuito de considerar a natureza probabilística destas incertezas, faz-se necessário identificar e definir estas variáveis como aleatórias no modelo de análise. Para consideração destas incertezas as normas atuais (NBR 6118/2014; NBR 8681/2003) indicam a aplicação de coeficientes de segurança, de forma a majorar os esforços e minorar as resistências.Com este procedimento atinge-se um risco aceitável para a estrutura. Para os Estados Limites de Serviço foram adotados coeficientes de segurança unitários, enquanto que para os Estados Limites Últimos foi adotado um fator de majoração de 1,4, tanto para cargas permanentes como para cargas variáveis.

Visto que, a resistência do concreto, a resistência das armaduras, os carregamentos aplicados às estruturas, assim como diversos outros parâmetros, são variáveis aleatórias, faz-se necessário um estudo de confiabilidade, visando diminuir ao máximo a probabilidade de falha da estrutura.

$\mathrm{O}$ principal objetivo da confiabilidade de estruturas é determinar a probabilidade de ocorrência de um cenário de falha na estrutura. Uma análise de confiabilidade permite, também, estimar a sensibilidade da segurança do projeto em relação às variáveis aleatórias consideradas no modelo. Essa informação é importante porque possibilita saber qual a influência de cada variável aleatória na probabilidade de falha.

O trabalho desenvolvido compreendeu basicamente três fases: i) o dimensionamento de cinquenta e quatro vigas de concreto protendido, conforme os critérios presentes na NBR 6118/2014 e NBR 8681/2003; ii) o desenvolvimento de um modelo para determinação da capacidade resistente devigas em concreto armado e protendido, aplicável a seções retangulares e $T$, o qual foi validado por meio da comparação entre o momento resistente calculado e resultados obtidos em ensaios experimentais realizados por Billet (1953), Feldmann (1954), Warwaruk (1957), Mattock, Yamazaki e Kattula (1971) e Tao e Du (1985); iii) a avaliação do índice de confiabilidade segundo o método de confiabilidade de primeira ordem (FORM - First Order Reliability Method), na qual foram utilizadas rotinas computacionais com base nos estudos de Low e Tang (2008).

\section{PROJETO DE VIGAS DE CONCRETO PROTENDIDO}

A fase de concepção de um projeto de engenharia deve englobar além da definição da estrutura, geometria da seção e especificação dos materiais, as verificações necessárias para o correto funcionamento da estrutura ao longo de sua vida útil.

A verificação da segurança deve seguir as disposições da NBR 8681, a metodologia encontra-se fundamentada em métodos semi-probabilísticos referenciados a Estados Limites, onde, após sua ocorrência a estrutura apresenta desempenho inadequado às finalidades da construção.

Com intuito de possibilitar a posterior comparação entre os resultados obtidos, foi definido um vão de cálculo $(l)$, duas seções transversais típicas (retangular e T), três valores para 
resistência característicaa compressão do concreto $\left(f_{c k}\right)$, três valores para resistência característicade ruptura a tração da armadura ativa $\left(f_{p t k}\right)$ e três relações $(r)$ entre carga permanente $\left(g_{k}\right)$ e carga variável $\left(q_{k}\right)$, mantendo-se constante a carga total, de forma que, foi necessário o dimensionamento de cinquenta e quatro vigas de concreto protendido. As seções típicas, o vão de cálculo e a carga total aplicada sobre as vigas são apresentadas na figura 5 .

Os valores definidos para a resistência característica do concreto foram $40 \mathrm{MPa}, 45 \mathrm{MPa}$ e $50 \mathrm{MPa}$. De acordo com Larrossaet al., que analisou o concreto de obras portuárias com $f_{c k}$ entre $40 \mathrm{MPa}$ e $50 \mathrm{MPa}$, o coeficiente de variação do concreto varia entre $4 \%$ e $12 \%$, assim, para o presente trabalho, adotou-se um valor fixo igual a $10 \%$ a favor da segurança. Para a resistência característica da armadura ativa utilizaram-se valores de $175 \mathrm{kN} / \mathrm{cm}^{2}, 190$ $\mathrm{kN} / \mathrm{cm}^{2}$ e $210 \mathrm{kN} / \mathrm{cm}^{2}$. Ainda em relação a protensão, foi definido para o projeto a utilização de protensão completa, nível 3, devido ao ambiente para o qual as vigas foram projetadas. $\mathrm{O}$ aço passivo considerado no projeto foi o CA-50. As relações entre carga permanente e carga variável, representadas por $r=$ $g_{k} /\left(g_{k}+q_{k}\right)$, foram definidas em 0,25 , 0,50 e 0,75 .

Para identificação das vigas dimensionadas foi utilizada a seguinte nomenclatura: tipo de seção da viga; resistência característica do concreto; resistência característica do aço utilizado na armadura ativa; relação entre cargas (índice $r$ ). Assim, para uma viga de seção retangular, com $f_{c k}=40 \mathrm{MPa}, f_{p t k}=$ $175 \mathrm{MPa}$ e $r=0,25$, tem-se a seguinte nomenclatura: VR.40.175.25.

A tabela 1 apresenta os dados variáveis utilizados para as vigas de seção retangular e as identifica conforme a nomenclatura proposta. Já a tabela 2 apresenta os dados respectivos às vigas de seção T.

\section{MODELO DE CÁLCULO PARA VERIFICAÇÃO DA CAPACIDADE RESISTENTE}

\subsection{Modelo de cálculo do momento resistente}

Visto que a verificação da capacidade resistente de vigas de concreto protendido objetiva a determinação do momento resistente (momento de ruptura), para uma dada seção, onde se conhece previamente as dimensões, armaduras e propriedades dos materiais aplicados, a análise dá-se no Estádio III. Para tal análise tem-se como hipóteses fundamentais as que se seguem: seções inicialmente planas e normais ao eixo da peça, assim permanecem em relação ao eixo deformado; existe aderência perfeita entre as armaduras, ativas e passivas, e o concreto que as envolve; a distribuição das deformações no regime de ruptura deve obedecer ao disposto na NBR 6118/2014 no que se refere aos domínios de deformações; o alongamento prévio deve ser incluído na deformação da armadura; a resistência à tração do concreto é desprezada na resistência aos esforços solicitantes; o diagrama tensão deformação é o parábola-retângulo, porém, permite-se a adoção do diagrama retangular simplificado; aplica-se o diagrama bilinear para a relação tensão-deformação da armadura ativa, conforme NBR 6118/2014; aplica-se o diagrama elastoplástico perfeito para a relação tensãodeformação da armadura passiva, conforme NBR 6118/2014.

$\mathrm{O}$ regime de ruptura, correspondente ao estado limite último, que ocorre por ruptura do concreto ou por deformação excessiva da armadura.Em se tratando de vigas sujeitas à flexão simples, a ruptura pode ocorrer nos domínios 2, 3 ou 4. As equações 1 e 2 apresentam o equilíbrio de forças e momentos, onde a primeira define a profundidade da linha neutra e a segunda o momento resistente, considerando-se a utilização do diagrama retangular simplificado. Porém a incógnita $x$ não pode ser obtida diretamente, visto que não é conhecido o Domínio de Deformação onde ocorre a ruptura.Assim, para determinação da profundidade da 
linha neutra aplicou-se um algoritmo interativo utilizando-se o método da bissecante.

$$
\begin{aligned}
\mathrm{M}_{\mathrm{R}}=\mathrm{R}_{\mathrm{pd}} *( & \left.\mathrm{d}_{\mathrm{p}}-0,4 * \mathrm{x}\right)+\mathrm{R}_{\mathrm{s} 2} \\
& *\left(0,4 * \mathrm{x}-d_{2}\right)+\mathrm{R}_{\mathrm{s} 1} \\
& *\left(0,4 * \mathrm{x}-\mathrm{d}_{1}\right)
\end{aligned}
$$$$
f(\mathrm{x})=R_{c c}(\mathrm{x})+\sum_{i=1}^{n} A_{s i} * \sigma_{s i}(\mathrm{x})+
$$

$\sum_{i=1}^{n} A_{p i} * \sigma_{p i}(\mathrm{x})$
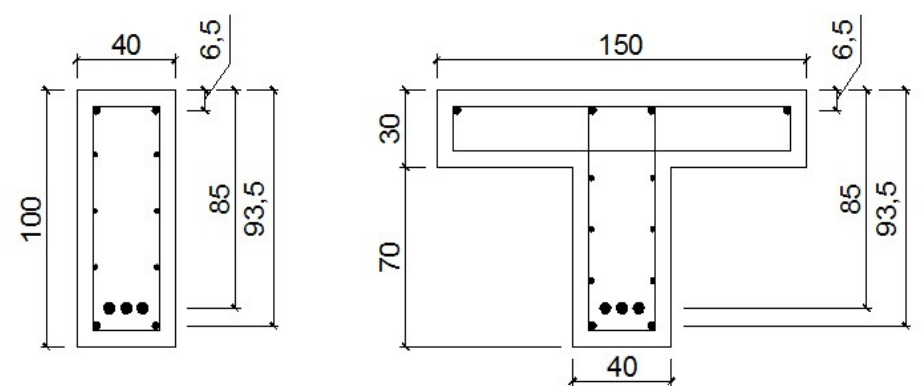

Dimensões em centímetros

$g$ - carga permanente

$q$ - carga variável

$S$ - carga total

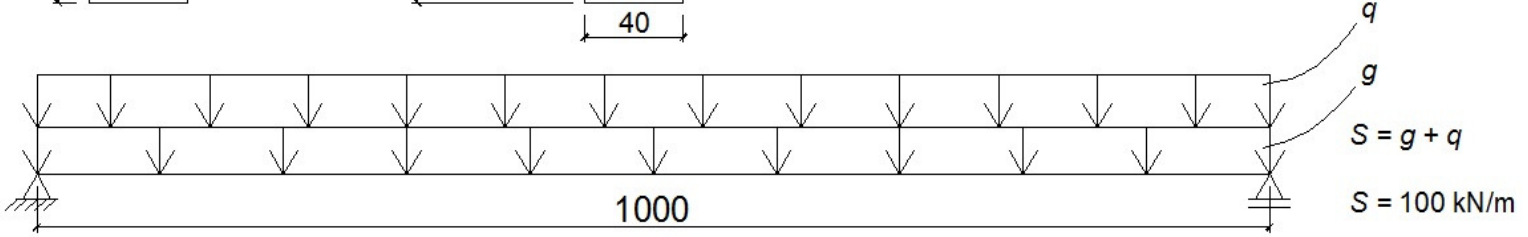

Figuras 5 - Seção transversal 1 e 2, vão de cálculo e cargas aplicadas. 
Tabela 1 - Dados variáveis - Vigas retangulares.

\begin{tabular}{|c|c|c|c|}
\hline Viga & $\begin{array}{c}\boldsymbol{f}_{\mathrm{ck}} \\
(\mathrm{MPa})\end{array}$ & $\begin{array}{c}\boldsymbol{f}_{\mathrm{ptk}} \\
\left(\mathrm{kN} / \mathrm{cm}^{2}\right)\end{array}$ & $\begin{array}{c}\boldsymbol{r} \\
\text { (\%) }\end{array}$ \\
\hline VR.40.175.25 & 40 & 175 & 25 \\
\hline VR.40.175.50 & 40 & 175 & 50 \\
\hline VR.40.175.75 & 40 & 175 & 75 \\
\hline VR.40.190.25 & 40 & 190 & 25 \\
\hline VR.40.190.50 & 40 & 190 & 50 \\
\hline VR.40.190.75 & 40 & 190 & 75 \\
\hline VR.40.210.25 & 40 & 210 & 25 \\
\hline VR.40.210.50 & 40 & 210 & 50 \\
\hline VR.40.210.75 & 40 & 210 & 75 \\
\hline VR.45.175.25 & 45 & 175 & 25 \\
\hline VR.45.175.50 & 45 & 175 & 50 \\
\hline VR.45.175.75 & 45 & 175 & 75 \\
\hline VR.45.190.25 & 45 & 190 & 25 \\
\hline VR.45.190.50 & 45 & 190 & 50 \\
\hline VR.45.190.75 & 45 & 190 & 75 \\
\hline VR.45.210.25 & 45 & 210 & 25 \\
\hline VR.45.210.50 & 45 & 210 & 50 \\
\hline VR.45.210.75 & 45 & 210 & 75 \\
\hline VR.50.175.25 & 50 & 175 & 25 \\
\hline VR.50.175.50 & 50 & 175 & 50 \\
\hline VR.50.175.75 & 50 & 175 & 75 \\
\hline VR.50.190.25 & 50 & 190 & 25 \\
\hline VR.50.190.50 & 50 & 190 & 50 \\
\hline VR.50.190.75 & 50 & 190 & 75 \\
\hline VR.50.210.25 & 50 & 210 & 25 \\
\hline VR.50.210.50 & 50 & 210 & 50 \\
\hline VR.50.210.75 & 50 & 210 & 75 \\
\hline
\end{tabular}

Tabela 2 - Dados variáveis - Vigas T.

\begin{tabular}{|c|c|c|c|}
\hline Viga & $\begin{array}{c}\boldsymbol{f}_{\mathrm{ck}} \\
(\mathrm{MPa})\end{array}$ & $\begin{array}{c}\boldsymbol{f}_{\text {ptk }} \\
\left(\mathbf{k N} / \mathbf{c m}^{2}\right)\end{array}$ & $\begin{array}{c}\mathbf{r} \\
\text { (\%) }\end{array}$ \\
\hline VT.40.175.25 & 40 & 175 & 25 \\
\hline VT.40.175.50 & 40 & 175 & 50 \\
\hline VT.40.175.75 & 40 & 175 & 75 \\
\hline VT.40.190.25 & 40 & 190 & 25 \\
\hline VT.40.190.50 & 40 & 190 & 50 \\
\hline VT.40.190.75 & 40 & 190 & 75 \\
\hline VT.40.210.25 & 40 & 210 & 25 \\
\hline VT.40.210.50 & 40 & 210 & 50 \\
\hline VT.40.210.75 & 40 & 210 & 75 \\
\hline VT.45.175.25 & 45 & 175 & 25 \\
\hline VT.45.175.50 & 45 & 175 & 50 \\
\hline VT.45.175.75 & 45 & 175 & 75 \\
\hline VT.45.190.25 & 45 & 190 & 25 \\
\hline VT.45.190.50 & 45 & 190 & 50 \\
\hline VT.45.190.75 & 45 & 190 & 75 \\
\hline VT.45.210.25 & 45 & 210 & 25 \\
\hline VT.45.210.50 & 45 & 210 & 50 \\
\hline VT.45.210.75 & 45 & 210 & 75 \\
\hline VT.50.175.25 & 50 & 175 & 25 \\
\hline VT.50.175.50 & 50 & 175 & 50 \\
\hline VT.50.175.75 & 50 & 175 & 75 \\
\hline VT.50.190.25 & 50 & 190 & 25 \\
\hline VT.50.190.50 & 50 & 190 & 50 \\
\hline VT.50.190.75 & 50 & 190 & 75 \\
\hline VT.50.210.25 & 50 & 210 & 25 \\
\hline VT.50.210.50 & 50 & 210 & 50 \\
\hline VT.50.210.75 & 50 & 210 & 75 \\
\hline
\end{tabular}




\subsection{Validação do modelo de cálculo}

Para aplicação do estudo de confiabilidade fez-se necessário a disposição de um modelo determinístico que representasse adequadamente a resposta do problema a ser estudado. Para suprir esta demanda desenvolveu-se um modelo de cálculo para capacidade resistente de vigas de concreto protendido, o qual pode ser aplicado também a vigas de concreto armado convencional.

Devido à necessidade de validação do modelo de cálculo procedeu-se a verificação de quarenta e uma vigas para as quais já se tinha o conhecimento do momento de ruptura, estes dados foram retirados da bibliografia existente e correspondem aos ensaios realizados anteriormente porBillet (1953), Feldmann (1954), Warwaruk (1957), Mattock, Yamazaki e Kattula (1971) e Tao e Du (1985). Observando-se o resultado obtido através do modelo determinístico frente aos resultados dos testes experimentais pode-se avaliar a precisão do modelo elaborado.

$\mathrm{Na}$ interpretação dos resultados obtidos deve-se considerar as limitações do modelo, visto que, as estruturas de concreto protendido apresentam um comportamento mecânico não-linear. Podem ser citadas como causas deste comportamento curvas tensãodeformação não-lineares dos materiais; a diferença entre as resistências a tração e à compressão do concreto; a aderência imperfeita entre o aço e o concreto adjacente; os fenômenos de retração e da fluência do concreto; a fissuração do concreto e a transmissão de esforços entre fissuras; o comportamento genérico da estrutura, etc. A análise completa da estrutura deveria levar em conta estes fatores, assim sendo, como o modelo numérico computacional desenvolvido não incluiu todas as variáveis presentes, devido a complexidade que seria exigida de tal modelo caso estas fossem consideradas, os resultados numéricos não acompanham exatamente os dados obtidos experimentalmente.
Acrescenta-se ainda que ensaios de laboratório também apresentam algumas incertezas, como por exemplo, o valor da carga atuante, as grandezas referentes aos materiais, condições de apoio e variações nas dimensões das peças estruturais. Os dados a serem utilizados nos modelos numéricos tendem a diferir, em maior ou menor grau, das propriedades existentes nas estruturas testadas em laboratório.Tal fato contribui para a divergência entre os valores medidos e calculados.

Conforme apresentado, a validação do modelo se deu pela relação entre o Momento de Ruptura Experimental $\left(M_{R, \text { exp }}\right)$ e o Momento Resistente Calculado $\left(M_{R, \text { calc }}\right)$, de forma que, a média dos resultados observados expressa o erro do modelo $\left(\eta_{\text {méd }}\right)$. O erro médio do modelo $\left(\eta_{\text {méd }}\right)$ e seu desvio padrão $\left(\sigma_{\eta}\right)$ são apresentados a seguir. Os resultados obtidos para o Momento de Ruptura Experimental e para o Momento Resistente são apresentados na tabela 3 . A nomenclatura utilizada para as vigas de validação do modelo é mesma dos autores dos experimentos.

$$
\begin{aligned}
& \eta=\frac{M_{R, \text { exp }}}{M_{R, \text { calc }}} \\
& \eta_{\text {méd }}=1,052 \\
& \sigma_{\eta}=0,076
\end{aligned}
$$

\section{ANÁLISE DE CONFIABILIDADE}

De acordo com Nowak e Collins (2000) muitas fontes de incerteza são intrínsecas ao projeto estrutural. Apesar do que muitas vezes se imagina os parâmetros da carga e da capacidade resistente dos elementos estruturais não são quantidades determinísticas. Elas são variáveis aleatórias, sendo assim, a segurança absoluta não pode ser alcançada. Consequentemente, as estruturas devem ser projetadas para servir a sua função com uma probabilidade finita de falha.

Tabela 3 - Momento Resistente Experimental e Momento de Ruptura Calculado. 


\begin{tabular}{|c|c|c|c|}
\hline Viga & $\begin{array}{l}M_{R, \exp } \\
(\mathrm{kN*m})\end{array}$ & $\begin{array}{l}M_{R \text {,calc }} \\
\left(\mathrm{kN}^{*} \mathrm{~m}\right)\end{array}$ & $\frac{M_{R, \text { exp }}}{M_{R, \text { calc }}}$ \\
\hline B1 & 49,98 & 46,049 & 1,085 \\
\hline B2 & 29,68 & 25,226 & 1,177 \\
\hline B3 & 15,36 & 12,872 & 1,193 \\
\hline B4 & 45,16 & 43,951 & 1,028 \\
\hline B5 & 55,60 & 53,684 & 1,036 \\
\hline B6 & 50,94 & 47,026 & 1,083 \\
\hline B7 & 72,94 & 77,557 & 0,941 \\
\hline B8 & 67,17 & 54,800 & 1,226 \\
\hline B9 & 47,72 & 47,467 & 1,005 \\
\hline B10 & 13,31 & 12,157 & 1,095 \\
\hline B11 & 47,22 & 45,071 & 1,048 \\
\hline B12 & 62,03 & 67,702 & 0,916 \\
\hline B13 & 48,02 & 45,198 & 1,063 \\
\hline B14 & 53,09 & 48,060 & 1,105 \\
\hline B15 & 48,31 & 47,966 & 1,007 \\
\hline B16 & 14,14 & 12,260 & 1,153 \\
\hline B17 & 45,89 & 45,833 & 1,001 \\
\hline B18 & 52,17 & 52,538 & 0,993 \\
\hline B19 & 71,56 & 73,274 & 0,977 \\
\hline B20 & 31,75 & 33,621 & 0,944 \\
\hline B21 & 34,40 & 34,005 & 1,012 \\
\hline B22 & 66,94 & 65,933 & 1,015 \\
\hline B23 & 79,98 & 83,915 & 0,953 \\
\hline B24 & 66,96 & 66,650 & 1,005 \\
\hline B25 & 50,17 & 47,249 & 1,062 \\
\hline B26 & 38,99 & 30,326 & 1,286 \\
\hline B27 & 70,14 & 73,456 & 0,955 \\
\hline B-28 & 30,70 & 26,056 & 1,178 \\
\hline B-29 & 50,63 & 46,377 & 1,092 \\
\hline B-30 & 17,64 & 15,678 & 1,125 \\
\hline B-31 & 44,93 & 45,150 & 0,995 \\
\hline B-32 & 65,31 & 69,177 & 0,944 \\
\hline B-33 & 43,36 & 41,489 & 1,045 \\
\hline J1 & 40,79 & 44,74 & 0,912 \\
\hline $\mathrm{J3}$ & 22,37 & 21,060 & 1,062 \\
\hline $\mathrm{J7}$ & 64,29 & 71,916 & 0,894 \\
\hline D1 & 35,00 & 27,744 & 1,262 \\
\hline D3 & 61,60 & 58,039 & 1,061 \\
\hline D10 & 71,40 & 60,004 & 1,190 \\
\hline RB1 & 93,44 & 94,840 & 0,985 \\
\hline TB1 & 109,93 & 105,484 & 1,042 \\
\hline
\end{tabular}

Para ilustrar a diferença entre quantidades determinísticas e aleatórias, consideremos as cargas impostas sobre um cais portuário. A carga sobre o cais, em qualquer momento depende de vários fatores, tais como o nível de operação do cais, os equipamentos presentes e o fluxo de carga. Tal fator varia diariamente em função da logística de operação dos portos. Diante destes fatores há incerteza sobre a carga total incidente sobre o cais, logo, a carga é uma variável aleatória.
Quanto ao conceito de "margem de segurança", este está associado aos estados limites últimos.

Tem-se como exemplo que, o modo de falha pode ser definido quando o momento solicitante exceder a capacidade resistente de uma viga de concreto protendido, sendo, a resistência "R" e a solicitação " $S$ ". Diante do exposto, a função de desempenho ou função de estado limite, é definida para este modo de falha como:

$$
g(R, S)=R-S
$$

O estado limite entre o desempenho desejado e indesejado ocorre quando $g=0 . \mathrm{Ou}$ seja, se $g>0$, a estrutura atua com o desempenho desejado (em segurança), se $g<0$, a estrutura encontra-se sob regime de ruptura (desempenho indesejado). A probabilidade de falha, $P f$, é igual à probabilidade de que ocorrerá ruína, ou seja, haverá o desempenho indesejável.

Pode-se expressar a probabilidade de falha em termos matemáticos como:

$$
P f=P(R-S<0)=P(g<0)
$$

Sendo $\mathrm{R}$ e $\mathrm{S}$ variáveis aleatórias, cada uma possui determinada função de densidade de probabilidade (PDF), temse ainda que, a quantidade de $R-S$ também é uma variável aleatória, com a sua respectiva função densidade de probabilidade.

Visto que, no presente estudo foi avaliada a capacidade resistente das vigas, a função de estado limite passa a ser escrita conforme equação 8.

$$
\begin{aligned}
& g(\mathrm{x})=M_{R}-M_{g}-M_{q}= \\
= & M_{R}-\frac{g * l^{2}}{8}-\frac{q * l^{2}}{8}
\end{aligned}
$$

O cálculo da probabilidade de sucesso ou da probabilidade de fracasso demanda que as funções densidade de probabilidade da resistência e solicitação ou que a função densidade de probabilidade conjunta sejam conhecidas, porém, via de regra tal informação não 
está disponível ou é de difícil obtenção devido à escassez de dados. Ainda assim, quando se dispõe das funções de densidade, a avaliação da probabilidade de falha geralmente requer integração numérica, o que pode tornar-se impraticável.

Quando não se dispõe das distribuições de probabilidade das variáveis, a confiabilidade pode ser obtida inteiramente como uma função do primeiro e do segundo momento (média e variância, respectivamente), pelo método de confiabilidade de Primeira Ordem e Segundo Momento ("First Order SecondMoment" - FOSM). Quando se conhecem as distribuições de probabilidade o Método de Confiabilidade de Primeira Ordem ("First Order Reliability Method" - FORM) ou a simulação de Monte Carlo podem ser utilizados (Ang e Tang, 1990).

A figura 6 apresenta a interpretação geométrica do índice de confiabilidade, onde $\beta$ é a distância mínima entre a origem e a superfície de falha e $\alpha_{i}^{*}$ são os cossenos diretores.

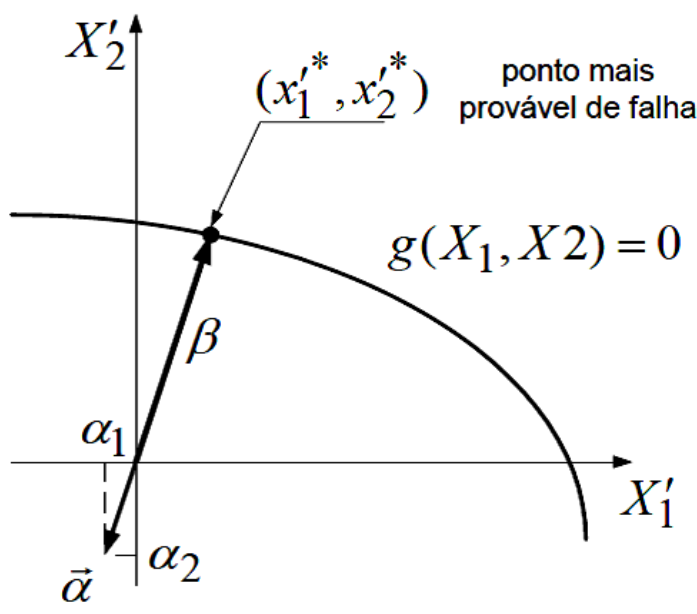

Figura 6- $\beta$ e cossenos diretores, representação geométrica.

As variáveis aleatórias consideradas para solução do problema encontram-se apresentadas na tabela 4, assim como o tipo de distribuição, média e desvio padrão.

\section{APLICAÇÃO E RESULTADOS}

\subsection{Entrada de dados resultados iniciais}

Definidos a geometria da seção, as características dos materiais empregados e a carga aplicada, partiu-se para o dimensionamento das vigas de concreto protendido, conforme critérios de projeto estabelecido nas normas vigentes. Diante da variabilidade dos parâmetros definidos necessitou-se projetar vinte e sete vigas de seção retangular e outras vinte e sete de seção $\mathrm{T}$, possibilitando assim a posterior análise paramétrica. Após o dimensionamento dos elementos passouse ao cálculo do índice de confiabilidade através do método FORM.

Tabela 4 - Variáveis aleatórias.

\begin{tabular}{|c|c|c|c|}
\hline Variável & $\mu_{\mathrm{x}}$ & $\mathrm{V}_{\mathrm{x}}=\sigma_{\mathrm{x}} / \mu_{\mathrm{x}}$ & Distribuição \\
\hline$f_{\mathrm{c}}$ & $\frac{f_{c k}}{(1-1,65 * V)}$ & 0,15 & Normal \\
\hline$f_{\mathrm{y}}$ & $\frac{f_{y k}}{(1-1,65 * V)}$ & 0,05 & Normal \\
\hline$f_{\mathrm{pt}}$ & $\frac{f_{p t k}}{(1-1,65 * V)}$ & 0,05 & Normal \\
\hline $\mathrm{d}_{\mathrm{s}}$ & $\mathrm{d}_{\mathrm{s}, \mathrm{nom}}$ & $\frac{0,5 \mathrm{~cm}}{\mathrm{~d}_{\mathrm{s}, \mathrm{nom}}}$ & Normal \\
\hline $\mathrm{d}_{\mathrm{p}}$ & $\mathrm{d}_{\mathrm{p}, \mathrm{nom}}$ & $\frac{1,0 \mathrm{~cm}}{\mathrm{~d}_{\mathrm{p}, \mathrm{nom}}}$ & Normal \\
\hline $\mathrm{g}$ & $\mathrm{g}_{\mathrm{nom}}$ & 0,1 & Normal \\
\hline $\mathrm{q}$ & $\mathrm{q}_{\mathrm{nom}}$ & 0,25 & $\begin{array}{c}\text { Valores } \\
\text { extremos tipo I }\end{array}$ \\
\hline$\eta$ & 1,052 & 0,072 & Normal \\
\hline
\end{tabular}

Para cálculo do Índice de Confiabilidade das vigas estudadas utilizou-se uma planilha eletrônica no software Excel, através da ferramenta de solução de equações não-lineares e minimização,Solver, tal ferramenta busca determinar um valor conforme restrições impostas na própria planilha. Trabalha-se com um grupo de células variáveis, que neste caso são os pontos de falha referentes a cada uma das variáveis aleatórias. A restrição imposta é a própria função estado limite, $g(x)=0$, admitida uma tolerância máxima. A tabela 5 apresenta os dados de entrada referentes às variáveis aleatórias para uma viga de 
seção retangular, resistência característica a compressão do concreto de $40 \mathrm{MPa}$, resistência característica de ruptura por tração da armadura ativa de $175 \mathrm{kN} / \mathrm{cm}^{2}$, resistência característica da armadura passiva de $50 \mathrm{kN} / \mathrm{cm}^{2}$ e relação de carga $r$ de 25\% (Viga VR.40.175.25).Além do disposto na tabela 5, devem ainda ser inseridos como dados de entrada as dimensões da seção, o vão de cálculo, o módulo de elasticidade dos aços utilizados, a deformação inicial da armadura ativa e a área de aço das armaduras (ativa e passiva).

Em função dos dados informados calcula-se a média e o desvio padrão da distribuição normal equivalente, sendo que, para primeira tentativa de convergência do modelo deve-se inserir os valores referentes aos pontosmédios das variáveis. Deve-se inserir ainda a matriz de correlação, que para o caso estudado, onde as variáveis são consideradas independentes, é uma matriz identidade $8 \times 8$. A tolerância utilizada, em todos os casos, para a convergência do sistema foi de $10^{-6}$.

Tabela 5 - Dados de entrada das variáveis aleatórias -VR.40.175.25.

\begin{tabular}{|l|c|c|c|c|c|c|}
\hline Distribuição & Variável & Unidade & Nominal & Média & Desv. Padrão & Coef. Var. \\
\hline Normal & $f_{\mathrm{c}}$ & $\mathrm{kN} / \mathrm{cm}^{2}$ & 4,000 & 5,316 & 0,797 & 0,150 \\
\hline Normal & $f_{\mathrm{y}}$ & $\mathrm{kN} / \mathrm{cm}^{2}$ & 50,000 & 54,496 & 2,725 & 0,050 \\
\hline Normal & $f_{\mathrm{pt}}$ & $\mathrm{kN} / \mathrm{cm}^{2}$ & 175,000 & 190,736 & 9,537 & 0,050 \\
\hline Normal & $\mathrm{d}_{\mathrm{s}}$ & $\mathrm{cm}$ & 93,500 & 93,500 & 0,500 & 0,005 \\
\hline Normal & $\mathrm{d}_{\mathrm{p}}$ & $\mathrm{cm}$ & 85,000 & 85,000 & 1,000 & 0,012 \\
\hline Normal & $\mathrm{g}$ & $\mathrm{kN} / \mathrm{m}$ & 25,000 & 25,000 & 2,500 & 0,100 \\
\hline Extvalue1 & $\mathrm{q}$ & $\mathrm{kN} / \mathrm{m}$ & 75,000 & 75,000 & 18,750 & 0,250 \\
\hline Normal & $\eta$ & adimensional & 1,052 & 1,052 & 0,076 & 0,072 \\
\hline
\end{tabular}

O momento resistente é determinado com base nos valores do ponto de falha para as variáveis aleatórias e nos valores de entrada para as demais variáveis, sobre o valor obtido é aplicado o erro do modelo $\eta^{*}$ (respectivo ao ponto de falha). O momento solicitante total $\left(M_{g}+M_{q}\right)$ é calculado tomando por base os valores do ponto de falha para as ações $g$ e $q$, e ainda, utilizando o valor de entrada para o vão de cálculo.

As tabelas 6 e 7 apresentam a área total de armadura de protensão, a deformação inicial da armadura ativa, e ainda, o valor do índice de confiabilidade. As vigas destacadas (*) tiveram sua armadura de protensão elevada para verificação do estado limite de serviço de formação de fissuras.

Tabela 6 - Vigas retangulares.

\begin{tabular}{|c|c|c|c|}
\hline Viga & $\begin{array}{c}\mathbf{A}_{\mathbf{p}} \\
\left(\mathbf{c m}^{\mathbf{2}}\right)\end{array}$ & $\boldsymbol{\beta}$ & $\boldsymbol{\varepsilon}_{\mathbf{p n}}$ \\
\hline VR.40.175.25* & 22,22 & 3,757 & 0,0045 \\
\hline VR.40.175.50 & 22,22 & 4,5789 & 0,0047 \\
\hline VR.40.175.75 & 23,23 & 6,186 & 0,0050 \\
\hline VR.40.190.25 & 20,20 & 3,7165 & 0,0050 \\
\hline VR.40.190.50 & 20,20 & 4,5315 & 0,0052 \\
\hline VR.40.190.75 & 22,22 & 6,3365 & 0,0052 \\
\hline VR.40.210.25 & 18,18 & 3,6969 & 0,0056 \\
\hline VR.40.210.50 & 18,18 & 4,5109 & 0,0059 \\
\hline VR.40.210.75 & 20,20 & 6,3496 & 0,0058 \\
\hline VR.45.175.25 & 21,21 & 3,6952 & 0,0047 \\
\hline VR.45.175.50 & 22,22 & 4,6774 & 0,0048 \\
\hline VR.45.175.75 & 23,23 & 6,3238 & 0,0051 \\
\hline VR.45.190.25* & 20,20 & 3,7978 & 0,0050 \\
\hline VR.45.190.50 & 20,20 & 4,6301 & 0,0053 \\
\hline VR.45.190.75 & 22,22 & 6,4802 & 0,0053 \\
\hline VR.45.210.25 & 18,18 & 3,7781 & 0,0056 \\
\hline VR.45.210.50 & 18,18 & 4,6111 & 0,0059 \\
\hline VR.45.210.75 & 20,20 & 6,4933 & 0,0058 \\
\hline VR.50.175.25 & 21,21 & 3,7404 & 0,0047 \\
\hline VR.50.175.50 & 22,22 & 4,7406 & 0,0048 \\
\hline VR.50.175.75 & 23,23 & 6,4342 & 0,0051 \\
\hline VR.50.190.25 & 19,19 & 3,681 & 0,0052 \\
\hline VR.50.190.50 & 20,20 & 4,6897 & 0,0053 \\
\hline VR.50.190.75 & 22,22 & 6,5944 & 0,0053 \\
\hline VR.50.210.25 & 17,17 & 3,6436 & 0,0059 \\
\hline VR.50.210.50 & 18,18 & 4,6668 & 0,0059 \\
\hline VR.50.210.75 & 20,20 & 6,6118 & 0,0059 \\
\hline
\end{tabular}

A área de armadura longitudinal passiva não foi considerada no dimensionamento a flexão e no cálculo da capacidade resistente, visto que,o momento solicitante foi suportado adequadamente pela armadura ativa longitudinal dimensionada, logo, $\mathrm{A}_{\mathrm{s}}=0$. Considerou-se o critério adotado em função da área de armadura protendida existente ser superior a área de armadura mínima calculada conforme NBR 6118 (2014). 
Tabela 7 - Vigas T.

\begin{tabular}{|c|c|c|c|}
\hline Viga & $\begin{array}{c}\mathbf{A}_{\mathbf{p}} \\
\left.\mathbf{( c m}^{\mathbf{2}}\right)\end{array}$ & $\boldsymbol{\beta}$ & $\boldsymbol{\varepsilon}_{\mathbf{p n}}$ \\
\hline VT.40.175.25 & 17,17 & 3,2455 & 0,0048 \\
\hline VT.40.175.50 & 18,18 & 4,2197 & 0,0050 \\
\hline VT.40.175.75 & 19,19 & 5,8362 & 0,0052 \\
\hline VT.40.190.25 & 15,15 & 3,094 & 0,0055 \\
\hline VT.40.190.50 & 16,16 & 4,0727 & 0,0057 \\
\hline VT.40.190.75 & 18,18 & 5,9844 & 0,0055 \\
\hline VT.40.210.25 & 14,14 & 3,1993 & 0,0059 \\
\hline VT.40.210.50 & 15,15 & 4,2141 & 0,0060 \\
\hline VT.40.210.75 & 16,16 & 5,8917 & 0,0063 \\
\hline VT.45.175.25 & 16,16 & 3,0432 & 0,0051 \\
\hline VT.45.175.50 & 18,18 & 4,2332 & 0,0050 \\
\hline VT.45.175.75 & 19,19 & 5,8569 & 0,0053 \\
\hline VT.45.190.25 & 15,15 & 3,1026 & 0,0054 \\
\hline VT.45.190.50 & 16,16 & 4,0858 & 0,0057 \\
\hline VT.45.190.75 & 18,18 & 6,0028 & 0,0055 \\
\hline VT.45.210.25 & 14,14 & 3,2081 & 0,0058 \\
\hline VT.45.210.50 & 15,15 & 4,2299 & 0,0061 \\
\hline VT.45.210.75 & 16,16 & 5,9099 & 0,0063 \\
\hline VT.50.175.25 & 16,16 & 3,0515 & 0,0051 \\
\hline VT.50.175.50 & 18,18 & 4,244 & 0,0050 \\
\hline VT.50.175.75 & 19,19 & 5,8712 & 0,0053 \\
\hline VT.50.190.25 & 15,15 & 3,1129 & 0,0055 \\
\hline VT.50.190.50 & 16,16 & 4,0963 & 0,0057 \\
\hline VT.50.190.75 & 18,18 & 6,0202 & 0,0056 \\
\hline VT.50.210.25 & 14,14 & 3,2187 & 0,0059 \\
\hline VT.50.210.50 & 15,15 & 4,2407 & 0,0061 \\
\hline VT.50.210.75 & 16,16 & 5,9243 & 0,0063 \\
\hline
\end{tabular}

\subsection{Avaliação paramétrica}

\subsubsection{Influência da Resistência Característica do Concreto}

Para as vigas retangulares analisadas, pode-se observar que a variação isolada do $f_{c k}$ possui baixa influência sobre o valor de $\beta$. É reconhecido o fato de que ao elevar-se o valor de $f_{c k}$ cresce linearmente a confiabilidade do sistema, porém, observou-se que tal elevação, nos estudos realizados, foi inferior a dois décimos no valor de $\beta$ para cada $5 \mathrm{MPa}$ elevados na resistência do concreto. A figura 7 ilustra o resultado obtido para um dos grupos de vigas estudados.

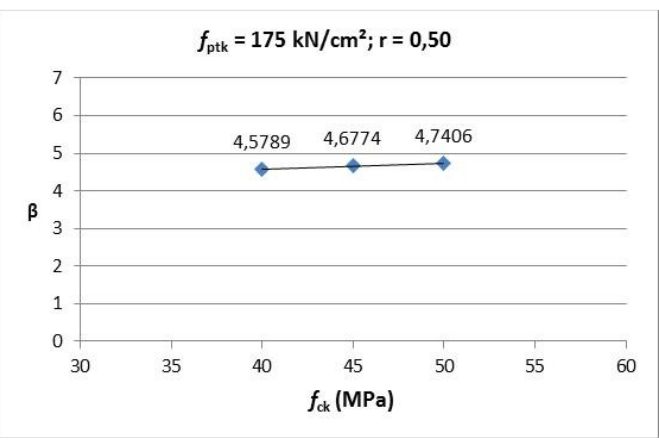

Figura 7 - Variação de $\beta$ em função de $f_{c k}\left(f_{p t k}=175 \mathrm{kN} / \mathrm{cm}^{2} ; r=0,50\right)$.

$\mathrm{Na}$ análise das vigas $\mathrm{T}$, observou-se a mesma tendência, porém, com variação ainda menor, pois para cada $5 \mathrm{MPa}$ acrescidos na resistência do concreto a elevação do índice de confiabilidade foi menor que cinco centésimos. A figura 8 ilustra o resultado obtido para um dos grupos de vigas estudados.

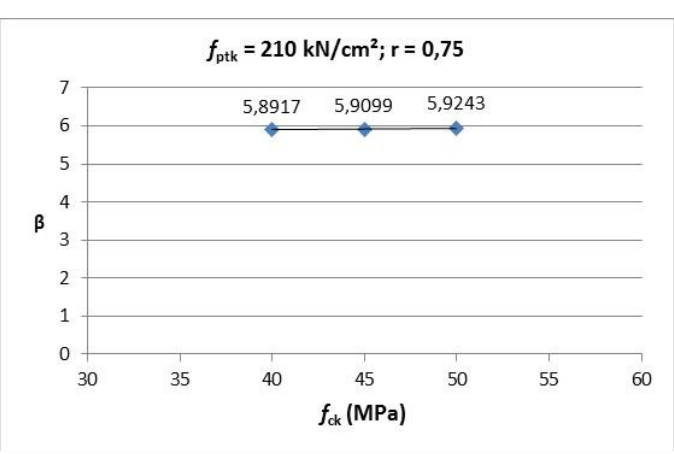

Figura 8 - Variação de $\beta$ em função de $f_{c k}\left(f_{p t k}=210 \mathrm{kN} / \mathrm{cm}^{2} ; r=0,75\right)$.

O comportamento observado se deve ao fato de que no estado limite último de flexão, para vigas de concreto protendido, a zona de concreto comprimido é bastante reduzida em relação à altura da seção, o que diminui a influência da resistência à compressão no cálculo do índice de confiabilidade.

\subsubsection{Influência da Resistência Característica da Armadura Ativa}

Ao analisar os resultados obtidos para as vigas de seção retangular, observou-se em alguns casos um pequeno crescimento da confiabilidade mediante a elevação da resistência do aço, porém, houve casos em que se verificou uma queda do índice de confiabilidade. Os 
comportamentos observados foram influenciados pela redução da área de aço, ocorrida após o aumento da resistência do mesmo, pois, o fato isolado do aumento da resistência, mantida a área de armadura ativa, tende a elevações consideráveis no valor do índice de confiabilidade.

A elevação da resistência da armadura ativa aumenta a confiabilidade do sistema. Porém, se ao elevar a resistência do aço o projetista optar por reduzir a área de armadura da seção, poderá a confiabilidade ficar prejudicada. Os comportamentos observadossão ilustrados nas figuras 9 e 10.

Para as vigas $\mathrm{T}$ projetadas foi observado o mesmo comportamento identificado para as vigas de seção retangular. A confiabilidade do sistema foi influenciada pela redução da armadura ativa, possibilitada pelo aumento da resistência do aço de protensão.As figuras 11 e 12ilustramo comportamento observado.

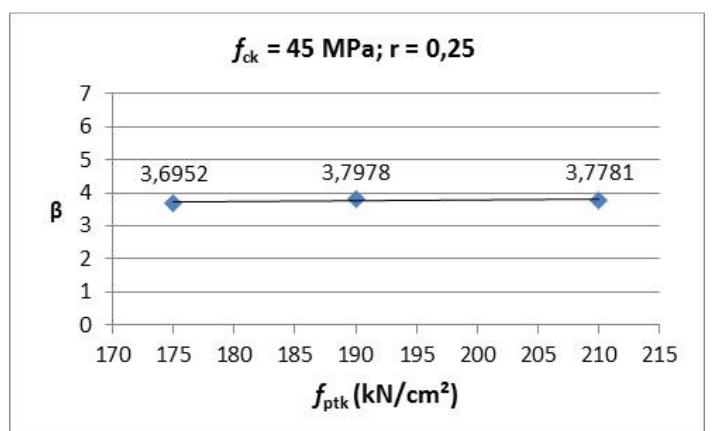

Figura 9 - Variação de $\beta$ em função de $f_{p t k}\left(f_{c k}=45 M P a ; r=0,25\right)$.

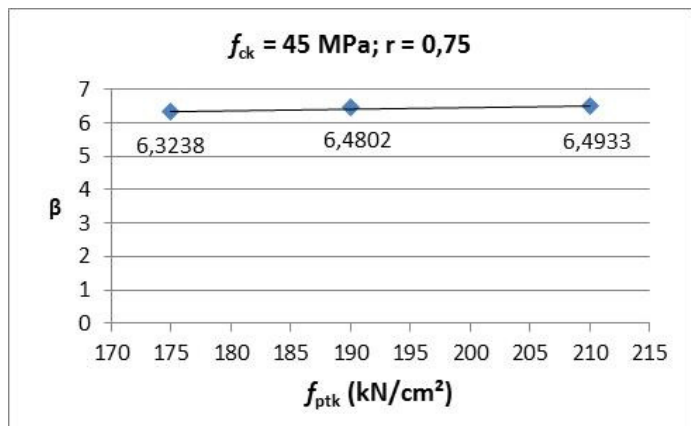

Figura 10 - Variação de $\beta$ em função de $f_{p t k}\left(f_{c k}=45 M P a ; r=0,75\right)$.

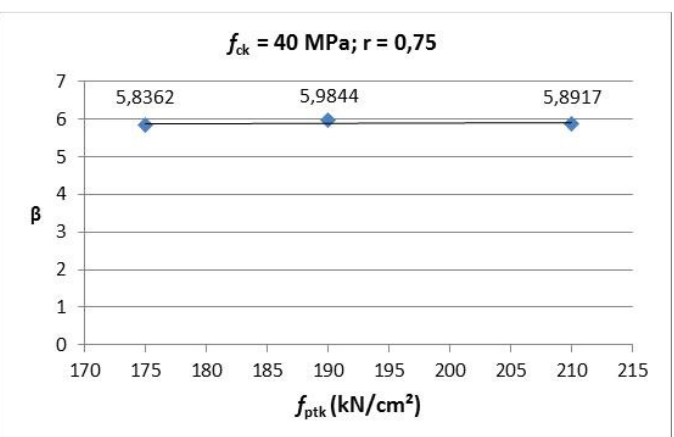

Figura 11 - Variação de $\beta$ em função de $f_{p t k}\left(f_{c k}=40 M P a ; r=0,75\right)$.

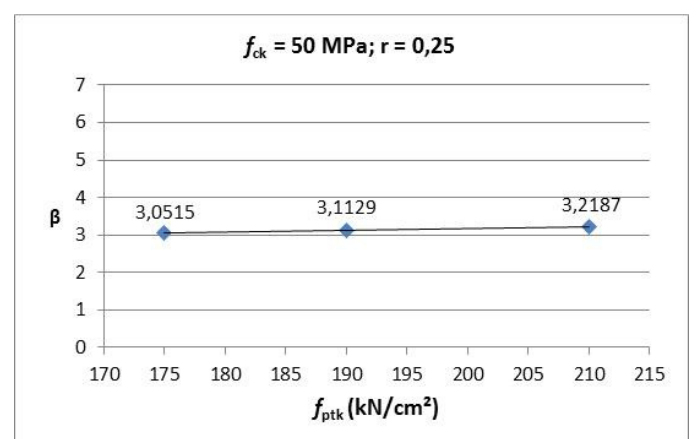

Figura 12 - Variação de $\beta$ em função de $f_{p t k}\left(f_{c k}=50 M P a ; r=0,25\right)$.

\subsubsection{Influência da Relação entre Carga Permanente e Acidental}

Para conclusão dos estudos paramétricos foi observado o índice de confiabilidade frente à variação da relação entre a carga permanente e a carga total, mantendo-se constante a carga total de $100 \mathrm{kN} / \mathrm{m}$, conforme já apresentado.

Nesta análise observando-se as vigas de seção retangular constatou-se que há uma tendência linear de crescimento do índice de confiabilidade mediante a elevação da carga permanente e consequente diminuição da carga variável. Pode-se observar ainda que, a partir do ponto em que as cargas se igualam há uma pequena mudança de inclinação na reta que representa a variação do índice de confiabilidade. Para valores da carga permanente menores que os da carga variável a confiabilidade cresce com menor intensidade, porém, para valores da carga permanente maiores que os da carga variável a confiabilidade cresce com maior intensidade, tal fato é observado na figura 13 . 


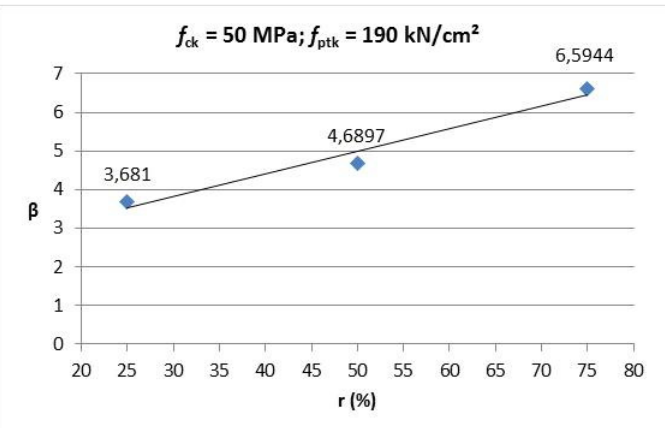

Figura 13 - Variação de $\beta$ em função de $r\left(f_{c k}=50 \mathrm{MPa} ; f_{p t k}=190 \mathrm{kN} / \mathrm{cm}^{2}\right)$

Ao avaliar-se o comportamento do índice de confiabilidade para as vigas $\mathrm{T}$, diante da variação da relação entre cargas, observou-se também a tendência de elevação da confiabilidade mediante o aumento da carga permanente $\mathrm{e}$ diminuição da carga variável, verificouse o mesmo comportamento em relação a inclinação da linha de tendência frente a proporção entre as duas cargas, para preponderância da carga variável constatou-se menor inclinação, enquanto que, para preponderância da carga permanente houve maior inclinação. A figura 14 ilustra o observado.

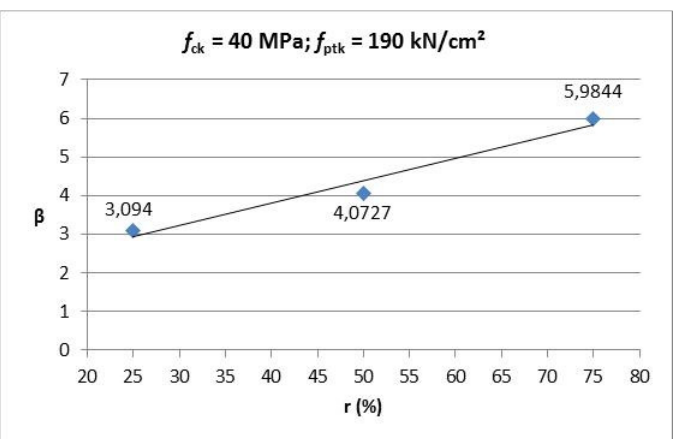

Figura 14 - Variação de $\beta$ em função de $r\left(f_{c k}=40 \mathrm{MPa} ; f_{p t k}=190 \mathrm{kN} / \mathrm{cm}^{2}\right)$

\section{CONCLUSÕES}

O estudo desenvolvido apresentou um modelo analítico para determinação do momento resistente em vigas de concreto protendido e posterior análise do índice de confiabilidade através do método FORM.

O modelo apresentado se mostrou adequado à verificação da capacidade resistente, uma vez que, os resultados estudados acompanharam os dados obtidos nos ensaios experimentais préexistentes. A determinação do erro médio e desvio padrão do modelo serviram de base para validação e posterior correção do momento resistente calculado.

A aplicação do método FORM através de planilha eletrônica, no software Excel, com uso da ferramenta Solver, mostrou-se bastante eficiente e prática para determinação do índice de confiabilidade.Visto que as normas brasileiras não especificam um índice de confiabilidade alvo foi adotado neste trabalho o valor referenciado por Nowak e Szerszen (2003), $\beta_{\text {alvo }}=3,5$. O índice de confiabilidade calculado para os elementos estruturais projetados esteve conforme o esperado acima do valor alvo, com exceção dos resultados obtidos para as vigas de seção $\mathrm{T}$ com relação entre cargas de 0,25. Nestes casos, onde houve a preponderância da carga variável sobre a carga permanente, necessita-se de uma revisão dos coeficientes de segurança apresentados nas normas brasileiras.

Os testes paramétricos, realizados permitiram a identificação dos parâmetros que afetam a confiabilidade do sistema, permitindo estabelecer uma relação da taxa de influência da variação de cada parâmetro sobre o valor do índice de confiabilidade.

Estudos sobre dois tipos de seções permitiram inferir sobre a relação de influência da geometria das vigas diante da confiabilidade do sistema. Ainda que seja esperada uma maior confiabilidade para vigas com seções de maior rigidez, como é o caso da utilização de uma seção $\mathrm{T}$ no lugar de uma seção retangular, há que se verificar a influência da alteração da área de armadura ativa quando da substituição de seção. Ao reduzir-se a área de armadura, tende-se a reduzir o Momento Resistente, causando a queda do Índice de Confiabilidade.

A avaliação do índice de confiabilidade frente à variação da resistência característica do concreto tornou possível concluir que a elevação de tal resistência, acarreta no aumento da confiabilidade, porém, verificou-se que esta variação positiva do índice de confiabilidade não é significativa frente aos outros parâmetros analisados. Este comportamento foi verificado para ambas 
seções estudadas. O comportamento observado se deve ao fato de que no estado limite último de flexão, para vigas de concreto protendido, a zona de concreto comprimido é bastante reduzida em relação à altura da seção, o que diminui a influência da resistência à compressão no cálculo do índice de confiabilidade.

A segunda relação paramétrica analisada foi a variação do índice de confiabilidade em função da alteração da resistência característica de ruptura por tração da armadura ativa, para tal parâmetro constatou-se elevação da confiabilidade mediante a utilização de aços com resistências maiores. Verificouse que para uma mesma seção transversal, com a simples elevação da resistência característica do aço, por exemplo, $175 \mathrm{kN} / \mathrm{cm}^{2}$ para $190 \mathrm{kN} / \mathrm{cm}^{2}$, o valor da confiabilidade se eleva, porém, se ao realizar esta alteração na resistência da armadura o projetista decidir diminuir a área de armadura, este fator irá tender a diminuir o nível de confiabilidade da estrutura, conforme observado em alguns casos. Visto que, o coeficiente de variação da resistência característica da armadura ativa foi mantido constante, em 0,05 , e ainda, que o desvio padrão desta variável cresce mediante o crescimento da própria variável, ao combinar-se estes fatos com a redução da área de aço de protensão pode-se provocar a queda da confiabilidade em alguns casos, como observado. Conclui-se que para uma mesma seção transversal, mantendo-se a área de armadura ativa e utilizando-se aços de resistência característica mais elevada a confiabilidade aumenta consideravelmente. Porém, sugere-se que diante do problema aqui levantado, queda da confiabilidade mediante a diminuição da área de protensão, quando da utilização de aços de mais alta resistência para vigas de concreto protendido, empregue-se um estudo mais detalhado de confiabilidade. Ainda que, a geometria da seção transversal influa no resultado da confiabilidade a proporção de elevação do índice de confiabilidade permaneceu constante para as seções estudadas.
O terceiro estudo paramétrico desenvolvido observou a relação do índice de confiabilidade com a variação entre carga permanente e carga variável, mantendo-se constante a carga total aplicada sobre as vigas. Observou-se para o referido parâmetro que há crescimento do nível de confiabilidade conforme a carga permanente é aumentada e a carga acidental diminuída.Tal fato se deve a relação entre o coeficiente de variação das duas parcelas de carga. O coeficiente de variação da carga permanente considerado neste estudo foi de 0,10 , já o da carga acidental foi de 0,25 , conforme já apresentado. Diante do exposto concluiu-se que a preponderância de cargas com menor coeficiente de variação, frente a outras cargas com maior coeficiente de variação, mantida a carga total, tende a elevar a confiabilidade do sistema. Verifica-se que para se obter um nível de confiabilidade ( $\beta$ )aproximadamente constante deveria ser adotado um fator de majoração maior para a carga variável, que possui coeficiente de variação maior.

Em função dos dados observados se verificou que a maior variação no índice de confiabilidade ocorreu frente à variação da relação entre carga permanente e carga total, porém, destacase a importância da análise conjunta das variáveis envolvidas no processo, bem como a relevância de uma análise detalhada da confiabilidade frente à variação da resistência característica de ruptura à tração da armadura ativa.

\section{REFERÊNCIAS}

ANG, A. H.; TANG, W. H..Probability concepts in engineering planning and design.Volume II: decision, riskandreliability. John Wiley\& Sons, 1990.

ASSOCIAÇÃO BRASILEIRA DE NORMAS TÉCNICAS. Projeto de estruturas de concreto - Procedimento: NBR-6118. Rio de Janeiro, 2014.

$\begin{array}{lccr}\text { ASSOCIAÇÃO } & \text { BRASILEIRA } & \text { DE } \\ \text { NORMAS } & \text { TÉCNICAS. Ações } & \text { e } \\ \text { segurança } & \text { nas estruturas } & -\end{array}$


Procedimento: NBR-8681.Rio de Janeiro, 2003.

BILLET, D. F..Study of prestressed concrete beams failing in flexure.University of Illinois, 1953.

FELDMAN, A..Bonded and unbonded prestressed concrete beams failing in flexure.University of Illinois, 1954.

LARROSSA, M. C.; REAL, M. V.; DIAS, C. R. R.; MAGALHÃES, F.C..Statistical analysis and conformity testing of concrete in port construction work.IBRACON Structures and Materials Journal, 2014.

LOW, B. K.; TANG, W. H..New FORM algorithm with example applications. Proc. the 4th Asian-Pacific Symposium on Structural Reliability and Its Applications APSSAR'08, Department of Civil Engineering,The Hong Kong University of Science \& Technology, Hong Kong, June 19-20, 2008, 221-226 Conference paper, 2008

MATTOCK, A. H.; YAMAZAKI, J.; KATTULA, B. T..Comparative study of concrete prestressed beams, with and without bond.ACI Journal, v.68, n. 2, February, 1971.

NOWAK, A. S.; COLLINS, K. R..Reliability of Structures.New York, McGraw-Hill, 2000.

TAO, X.; DU, G..Ultimate stress of unbonded tendons in partially prestressed concrete beams. PCI Journal 30(6): 72-91.1985

WARWARUK, J..Strength in flexure of bonded and unbonded prestressed concrete beams. Universityof Illinois, 1957. 